\title{
ATUAÇÃO DE ENFERMEIROS NA ESTRATÉGIA DE SAÚDE DA FAMÍLIA EM UM MUNICÍPIO DE MINAS GERAIS
}

\author{
ACTION OF NURSES FROM THE FAMILY HEALTH STRATEGY \\ IN A CITY OF MINAS GERAIS
}

\begin{abstract}
Anilson Augusto Firmino', Mariana Cristina Moraes², Priscila Elídia Alves Nascimento², Sônia Maria Alves de Paiva ${ }^{3}$, Cristiane Aparecida Silveira ${ }^{3}$
\end{abstract}

\section{RESUMO}

Objetivo: caracterizar a atuação dos enfermeiros das Estratégias de Saúde da Família em um município de Minas Gerais. Método: estudo exploratório-descritiva, de abordagem qualitativa. Foram entrevistados 15 enfermeiros utilizando um roteiro de entrevista semi-estruturado incluindo questões relativas as ações que realiza regularmente. A coleta de dados foi realizada durante o segundo semestre de 2014. Resultados: perfil predominantemente feminino, jovens, 6 (40,0\%) possuíam tempo de exercício profissional de até 1 ano, 12 (80,0\%) possuíam especialização. As atividades realizadas foram compatíveis com as atribuições dos enfermeiros segundo o a Politica Nacional de Atenção Básica do Ministério da Saúde. As dificuldades mencionadas para o desenvolvimento das atividades propostas foram a estrutura física, a população adscrita, maior que o preconizado, a falta de informação da população, recursos materiais, humanos e financeiros deficientes e questões relativas à gestão deficiente. Conclusão: é necessário refletir sobre a formação do enfermeiro, buscando uma qualificação para melhor desenvolvimento de suas competências, melhorar a gestão dos recursos, bem como investir na educação em saúde da população sobre o modelo de saúde adotado.

Descritores: Estratégia Saúde da Família; Enfermagem; Sistema Único de Saúde.

\section{ABSTRACT}

Objective: characterizing the performance of the nurses from the Family Health Strategy in a municipality of Minas Gerais. Methods: an exploratory descriptive study of a qualitative approach. There were interviewed 15 nurses using a semi-structured interview guide including questions concerning the actions that performs regularly. Data collection was carried out during the second half of 2014. Results: a predominantly female profile, young, $6(40,0 \%)$ had professional exercise time until 1 year, $12(80,0 \%)$ had expertise. The activities were compatible with the nurses' assignments according to the National Basic Care Policy of the Ministry of Health. The mentioned difficulties for the development of the proposed activities were the physical structure, the ascribed population, more than the stated, the lack of information of the population, material resources, human and financial issues relating to the disabled and poor management. Conclusion: it is necessary to reflect about nurse's formation, seeking a qualification to better develop their skills, improve the management of resources, as well as investing on health education of the population about the health model adopted.
${ }^{1}$ Graduado em Enfermagem pela Pontifícia Universidade Católica de Minas Gerais (PUC Minas), Poços de Caldas, MG, Brasil.

${ }^{2}$ Graduada em Enfermagem pela Pontifícia Universidade Católica de Minas Gerais (PUC Minas), Poços de Caldas, MG, Brasil.

${ }^{3}$ Doutora em Enfermagem pela Escola de Enfermagem de Ribeirão Preto da USP (EERPUSP), São Paulo, SP, Brasil.

Descriptors: Family Health Strategy; Nursing; Unified Health System. 


\section{Introdução}

A Estratégia de Saúde da Família (ESF) como forma de reorganização da atenção primária busca fortalecer-se como porta de entrada do Sistema Único de Saúde (SUS), reafirmando os princípios e diretrizes propostas pelas novas políticas de saúde pública, desconstruindo o paradigma do modelo de atenção à saúde curativista, hospitalocêntrico e pouco resolutivo ${ }^{1 ;}$; visando ações de promoção, proteção e recuperação da saúde de forma integral e contínua, por intermédio de um empoderamento da população e ações de educação em saúde, incentivando o autocuidado em relação à sua saúde?

A estratégia nasceu na tentativa de repensar os padrões de pensamento e comportamento dos profissionais e cidadãos brasileiros, até então vigentes. Envolve médicos, enfermeiros, técnicos de enfermagem, odontólogos e Agentes Comunitários de Saúde (ACS), busca discutir e ampliar o tradicional modelo sanitário médico-curativista, para a compreensão de uma abordagem coletiva, multi e interprofissional, centrada na família e na comunidade, inserida em seu contexto real e concreto².

A ESF traz propostas para mudar toda a antiga concepção de atuação dos profissionais de saúde, saindo da medicina curativa e passando a atuar na integralidade da assistência, tratando o indivíduo como sujeito dentro da sua comunidade socioeconômica e cultural, considerando essa dimensão globalizante ${ }^{1}$.

Neste cenário, a composição das unidades de saúde, configuradas dentro de um modelo de ESF deve incluir no mínimo, um médico clínico geral, um enfermeiro, um auxiliar/técnico de enfermagem e de quatro a seis ACS, cada qual com suas atribuições específicas e com uma atuação interdisciplinar ${ }^{3}$.

O modelo de assistência da ESF constitui um desafio para o enfermeiro que como participante da equipe de saúde, deve considerar o envolvimento do seu agir com os aspectos sociais, políticos, econômicos e culturais relevantes para o processo de transição e consolidação do novo modelo da assistência à saúde ${ }^{4}$.

O campo de atuação do enfermeiro dentro deste contexto exige do mesmo, qualificação e perfil diferenciado, com pleno conhecimento de suas atribuições, como forma de garantir a efetividade de suas ações.

O enfermeiro deve ter conhecimento dos conceitos de família, saúde da família e interação familiar, para prestar uma assistência, considerando o aspecto estrutural, quanto organizacional da assistência em saúde pública, para que assim possa oferecer um cuidado de maior qualidade e resolutividade ${ }^{5}$.

O enfermeiro desempenha relevante papel, resgatando o vínculo de atenção enfermeiro/família, na busca de contribuir para melhoria da qualidade de saúde e de vida do indivíduo no ambiente familiar. No tocante as suas atribuições, o enfermeiro deve executar ações de assistência básica de vigilância epidemiológica e sanitária à criança, ao adolescente, à mulher, ao trabalhador e à terceira idade. Também deve atuar como instrutor/supervisor dos agentes comunitários de saúde; no gerenciamento do pessoal de enfermagem e da Unidade de Saúde, além de participar do Conselho de Saúde do município. Ao enfermeiro da ESF cabe a gerencia da assistência de enfermagem, devendo 0 enfermeiro ser o gerador de conhecimento, através do desenvolvimento de competências, introduzindo inovações à equipe, definindo responsabilidades. Além disso, a assistência de enfermagem prestada às famílias deve ser coerente com as condições socioeconômicas, culturais e ambientais da família e comunidade 4 .

As principais dificuldades encontradas pelos enfermeiros da ESF, tais como: assistência predominantemente curativa; a maioria dos enfermeiros revela desconhecimento sobre seu o papel gerencial, dificuldades em desenvolver a autonomia profissional, postura inadequada de "coleguismo" e o fazer nem sempre associado ao "saber"; os sujeitos relatam dificuldades no dia a dia relacionados com as diferentes personalidades e opiniões divergentes entre os membros da equipe, informam haver um despreparo dos ACS e do médico em integrar os princípios da Estratégia, a dificuldade em articular uma assistência social para intervir nos problemas sociais da população e o uso pelos políticos para benefícios particulares ${ }^{6}$.

É importante ressaltar a ESF como uma estratégia de fomento à participação da população. $O$ enfermeiro deve, junto com a equipe, promover uma nova relação entre os sujeitos, onde tanto os profissionais quanto os usuários possam ser produtores e construtores de um viver mais saudável ${ }^{7}$. 
Considerando que a atuação da enfermagem na saúde da família vem se consolidando na prática e na experiência adquirida pelos profissionais de saúde na saúde coletiva e que enfermagem na $E S F$, para construir um trabalho em equipe em uma nova concepção do processo de trabalho e que o enfermeiro precisa delinear cada vez mais e melhor 0 seu campo de atuação profissional e desenvolver o seu projeto político-legal, coerente com os princípios e diretrizes do SUS, bem como com as diretivas da ESF, justifica-se a presente investigação.

Diante do exposto, este estudo tem como questão norteadora: Como se caracteriza a atuação dos enfermeiros das Estratégias de Saúde da Família em um município de Minas Gerais? Para tanto, objetivou-se caracterizar a atuação dos enfermeiros das Estratégias de Saúde da Família em um município de Minas Gerais.

\section{Metodologia}

Trata-se de uma pesquisa exploratório-descritiva, de abordagem qualitativa desenvolvida em 15 unidades de ESF, ou seja a totalidade das unidades, localizadas em um município de Minas Gerais. O projeto foi autorizado pela Secretaria Municipal de Saúde e pelo Comitê de Ética em Pesquisa (4122.0.000.213), seguindo todas as Diretrizes da Resolução 466/128.

Os critérios de inclusão foram: ser enfermeiro da ESF há pelo menos seis meses e aceitar participar do estudo. Os convites foram encaminhados a todas as ESF do município. Depois foi feito contato telefônico com os mesmos para confirmar a participação. Foi agendada uma reunião para orientações sobre os objetivos do trabalho e a confidencialidade de suas respostas. Todos os enfermeiros das ESF preenchiam os critérios da pesquisa (pelo menos 6 meses de experiência na ESF e aceitaram participar). Após aceitaram participar do estudo e assinaram o Termo de Consentimento Livre e Esclarecido (TCLE).

As entrevistas foram realizadas por meio da utilização de um roteiro de entrevista semiestruturado incluindo questões relativas à sua participação na elaboração de protocolos para as ações da equipe e ações que realiza regularmente. As entrevistas foram conduzidas pelas próprias pesquisadoras durante o segundo semestre de 2014. A questão norteadora foi: Como se caracteriza a atuação dos enfermeiros das Estratégias de Saúde da Família em um município de Minas Gerais?

O referencial teórico considerado foram as atividades desenvolvidas pelo enfermeiro da ESF constantes na Portaria $n^{0}$ 2.488, de 21 de outubro de 2011 que aprova a Política Nacional de Atenção Básica, estabelecendo a revisão de diretrizes e normas para a organização da Atenção Básica, para a Estratégia Saúde da Família (ESF) e o Programa de Agentes Comunitários de Saúde (PACS)9.

As variáveis consideradas foram: idade, tempo de exercício profissional, carga horária, qualificação na ESF, experiência anterior em saúde pública.

Para análise e interpretação dos dados, utilizou-se a análise de conteúdo ${ }^{10}$. Após a categorização das falas, procedeu-se, então, à inferência por meio dos dados obtidos e utilizando como base teórica as atribuições do enfermeiro na ESF de acordo com a Portaria 2488/11 do Ministério da Saúde que estabelece as Diretrizes e normas para a organização da Atenção Básica, para a Estratégia Saúde da Família9 .

Para a diferenciação dos sujeitos, bem como preservação de sua identidade, os informantes foram identificados com a letra $\mathrm{E}$ acompanhada de numeral arábico, o qual indica a ordem em que foram realizadas as entrevistas. Não foram feitas correções de linguagem na fala dos sujeitos.

\section{Resultados}

Com relação aos dados sobre sexo, faixa etária e tempo de formação, segundo as respostas dos questionários, foram quantificados e apresentados na Tabela 1. 
Tabela 1 - Distribuição dos profissionais de enfermagem na ESF, segundo dados biopsicossociais. Minas Gerais, 2014.

\begin{tabular}{|c|c|c|c|}
\hline \multirow{2}{*}{\multicolumn{2}{|c|}{ Variáveis }} & \multicolumn{2}{|c|}{ Total } \\
\hline & & f & $\%$ \\
\hline \multirow[t]{3}{*}{ Sexo } & Masculino & 1 & 6,67 \\
\hline & Feminino & 14 & 93,33 \\
\hline & Total & 15 & 100 \\
\hline \multirow[t]{7}{*}{ Idade } & 20 a 25 anos & 7 & 46,67 \\
\hline & 25 a 30 anos & 5 & 33,33 \\
\hline & 30 a 35 anos & 2 & 13,33 \\
\hline & 35 a 40 anos & - & - \\
\hline & 40 a 50 anos & 1 & 6,67 \\
\hline & Acima de 60 anos & - & \\
\hline & Total & 15 & 100 \\
\hline \multirow[t]{6}{*}{ Tempo de exercício profissional } & O a 1 ano & 6 & 40,0 \\
\hline & 1 a 2 anos & 3 & 20,0 \\
\hline & 2 a 5 anos & 4 & 26,67 \\
\hline & 5 a 10 anos & - & - \\
\hline & Acima de 10 anos & 2 & 13,33 \\
\hline & Total & 15 & 100 \\
\hline
\end{tabular}

Fonte: Dados da Pesquisa

Pôde-se constatar o predomínio do sexo feminino: 14 (93,33\%) entre os profissionais, reproduzindo a característica histórica da enfermagem, profissão exercida quase que exclusivamente por mulheres, desde os seus primórdios.

Os dados obtidos referentes à faixa etária dos sujeitos da pesquisa foram: $12(80,0 \%)$ entre 20 a 30 anos, dois $(13,33 \%)$ de 30 a 35 anos e um $(6,67 \%)$ de 40 a 50 anos. 0 tempo de trabalho nas unidades foi $6(40,0 \%)$ de 0 a 1 ano, $3(30,0 \%)$ de 1 a 2 anos e $4(26,67 \%)$ de 2 a 5 anos.

Com relação à formação para atuar na atenção primária, constatou-se que, apenas $3(20,0 \%)$ dos enfermeiros tinham participado de capacitação em ESF, com carga horária entre 48 e 80 horas;

Quanto a especialização: $12(80,0 \%)$ profissionais tinham Especialização na Saúde da Família, sendo que 5 $(33,33 \%)$ possuíam curso de especialização de 360 horas, 1 (6,67\%) de 390 horas, 2 (13,33\%) de 400 horas, $1(6,67 \%)$ de 460 horas, $2(13,33 \%)$ de 480 horas e $1(6,67 \%)$ de 540 horas.

Com relação ao exercício em outra atividade, 2 (13,33\%) enfermeiros têm jornada dupla de trabalho em serviços de Urgência e Emergência. $O$ fato da jornada de trabalho do enfermeiro na ESF ser de quarenta horas semanais e complementar essa carga, com outra; demanda um desgaste físico e psíquico, que pode predispor o trabalhador a doenças ocupacionais e comprometer a assistência de enfermagem ao usuário.

Em relação à disponibilidade de dedicação exclusiva ao PSF, apontam que $32,1 \%$ de seus profissionais trabalham em outros locais, ou seja, acumulam atividades profissionais ( $100 \%$ dos médicos, $67 \%$ dos enfermeiros e $17 \%$ dos ACS ${ }^{11}$. Isso acaba afetando não só a qualidade da atenção, mas a própria saúde dos trabalhadores.

Quanto aos protocolos para o desenvolvimento de suas atividades dentro da unidade, 11 (93,33\%) dos enfermeiros admitiram seguir as Linhas Guias do Ministério da Saúde. Entretanto, somente $3(20,0 \%)$ participaram da elaboração dos protocolos locais segundo estas diretrizes.

A Tabela 2 apresenta as principais atividades realizadas pelos enfermeiros. 
Tabela 2 - Relatos dos profissionais sobre quais as ações realizadas regularmente nas ESF. Minas Gerais, 2014.

\begin{tabular}{|c|c|c|}
\hline \multirow[t]{2}{*}{ Categorias } & \multicolumn{2}{|c|}{ Total } \\
\hline & $\mathbf{f}$ & $\%$ \\
\hline Consultas & 7 & 46,67 \\
\hline Exame Papanicolau & 4 & 26,67 \\
\hline Visita Domiciliar & 8 & 53,33 \\
\hline Educação Permanente com a equipe & 7 & 46,67 \\
\hline $\begin{array}{c}\text { Atividades com a população (grupos de educação em } \\
\text { saúde, caminhadas, eventos comemorativos) }\end{array}$ & 10 & 66,67 \\
\hline Puericultura & 3 & 20,0 \\
\hline Gestão & 8 & 53,33 \\
\hline Reunião de equipe & 3 & 20,0 \\
\hline Acolhimento da população para consulta & 7 & 46,67 \\
\hline $\begin{array}{l}\text { Procedimentos técnicos (curativos, administração de } \\
\text { injeções e inalação, entrega de medicamentos). }\end{array}$ & 4 & 26,67 \\
\hline
\end{tabular}

Fonte: Dados da Pesquisa

Os dados das falas permitiram constatar que as ações mencionadas pelos enfermeiros incluem consultas de enfermagem, exame de Papanicolau, visita domiciliar, educação permanente para a equipe, ações junto à população, puericultura, gerenciamento em enfermagem, acolhimento, reunião de equipe, procedimentos técnicos (curativos, administração de medicamentos, dentre outros).

Bom, (...) o meu tempo fica completamente tomando conta das atividades como (...) acolhimento (...) atendimento de crianças e mulheres, grupos de hipertensos e diabéticos (E3).

Tabela 4 - Relatos dos profissionais sobre a maior dificuldade no desenvolvimento dessas atividades. Minas Gerais, 2014.

\begin{tabular}{c|c|c}
\hline Categorias & \multicolumn{2}{|c}{ Total } \\
& f & $\%$ \\
\hline Estrutura física & 5 & 33,33 \\
\hline $\begin{array}{c}\text { População adscrita, maior que o } \\
\text { preconizado pelo MS }\end{array}$ & 4 & 26,67 \\
\hline Falta de informação da população & 3 & 20,0 \\
\hline Recursos materiais & 2 & 13,33 \\
\hline Recursos humanos & 2 & 13,33 \\
\hline Gestão & 2 & 13,33 \\
\hline Recursos financeiros & 1 & 6,67 \\
\hline
\end{tabular}

Fonte: Dados da Pesquisa

Quanto às dificuldades para realização dessas atribuições dentro da unidade, as respostas identificaram a estrutura física; o tamanho da população adscrita, os recursos (materiais, humanos e financeiros); a gestão e a falta de informação por parte da população quanto à finalidade da ESF. 
A grande dificuldade que enfrento é a falta de compreensão (...) as pessoas (...) não entendem que aqui não é pronto-atendimento (...) que aqui tem um trabalho com objetivos a longo prazo (...) (E7).

Outra dificuldade (...) é a questão que esta unidade atende muita gente (...) é três em uma (...) até que as outras sejam implantadas a gente fica muito sobrecarregado (E13).

\section{Discussão}

O perfil jovem da população é semelhante ao disposto na literatura ${ }^{12}$. O setor de maior empregabilidade é o setor público, sobretudo a ESF, que tem proporcionado maior oportunidade para os egressos, normalmente jovens ${ }^{12}$.

O Ministério da Saúde (MS) tem preconizado a capacitação como instrumento de atualização permanente, caracterizando os aspectos gerais da Atenção Primária à Saúde. O MS reconhece e valoriza a formação dos trabalhadores como um componente para o processo de qualificação da força de trabalho no sentido de contribuir decisivamente para a efetivação da política nacional de saúde ${ }^{9}$.

A falta de profissionais com formação adequada e com perfil generalista, para atuar na ESF, passou a ser um grande obstáculo para sua consolidação. Dessa forma, o MS assumiu institucionalmente, através do Departamento de Gestão da Educação na Saúde a responsabilidade de reorientar ações de formação dos profissionais de saúde, que tivessem como foco novos modos de cuidar e ensinar em saúde ${ }^{13}$.

É consenso entre os gestores e trabalhadores do SUS, em todas as esferas de governo, de que a formação, o desempenho e a gestão dos recursos humanos afetam, profundamente, a qualidade dos serviços prestados e 0 grau de satisfação dos usuários; destacando-se, assim, a formação e educação dos profissionais no processo saúde-doença com enfoque na saúde da família, como importante desafio para o êxito do modelo sanitário proposto ${ }^{13}$.

O enfermeiro integra a equipe a equipe de saúde e assume a gerencia da ESF. Deve ser capazes de planejar, organizar, desenvolver e avaliar ações que respondam às necessidades da comunidade, na articulação com os diversos setores envolvidos na promoção da saúde e para tanto, necessita de conhecimento e preparo para assumir essa competência ${ }^{14}$.

As Linhas-Guias têm por finalidade a organização sistêmica dos serviços, com objetivo de racionalização dos recursos e otimização do trabalho, mantendo ou melhorando a qualidade do serviço. São elas: atenção ao pré-natal, ao parto e ao puerpério; atenção às crianças, ao neonato, aos adolescentes, aos idosos; atenção à tuberculose, hanseníase, hipertensão, diabetes, saúde mental, saúde bucal e HIVIAIDS ${ }^{13}$. É importante que os profissionais da prática atuem na elaboração dos protocolos, para que desenvolvam suas competências e saberes, ampliando sua prática profissional e garantindo melhor estrutura, organização e funcionamento dos sistemas e serviços da ESF ${ }^{15}$.

Os dados das falas permitiram constatar que as ações mencionadas pelos enfermeiros estão de acordo com as atribuições propostas pela Política Nacional de Atenção Básica ${ }^{11}$. A coleta de exame Papanicolau constitui uma importante ação na prevenção e promoção da saúde da mulher, enquanto a visita domiciliar é um instrumento fundamental na prestação da assistência à saúde, do indivíduo, família e comunidade, possibilitando conhecer a realidade do cliente e sua família. Permite avaliar, desde as condições ambientais e físicas em que vivem o indivíduo e sua família, até assistir os membros do grupo familiar, além de aplicar medidas de controle nas doenças transmissíveis ou parasitárias ${ }^{11}$.

A consulta de enfermagem, como prática clínica, é reconhecida como importante pelo enfermeiro, e é vista no âmbito do SUS, como uma importante ferramenta para aumentar a qualidade da assistência à saúde da população ${ }^{11}$. A consulta de enfermagem deve contribuir para superar o processo de atendimento sob a ótica do modelo biomédico; é considerada uma atividade autônoma, pela qual o profissional tem a possibilidade de estabelecer laços consistentes com o cliente. Pode fornecer dados relevantes, não somente a respeito de sua condição física, como abrir espaço para outras dimensões merecedoras de atenção; entretanto, permanece, ainda na lógica da atenção clínica individual, curativa ${ }^{16}$.

A educação em saúde deve focar em estratégias de promoção à saúde que ampliem o foco dos cuidados para além da preocupação com a doença, podendo resgatar aspectos importantes para estimular a autonomia dos indivíduos e transformar a realidade social. Devem estimular a organização comunitária e a autonomia das famílias, tendo em vista que o modelo assistencial proposto é o de privilegiar a promoção da saúde, se baseia no encorajamento e apoio para que as pessoas e grupos sociais assumam maior controle sobre os determinantes de saúde e provoquem mudanças de hábitos de vida dos usuários ${ }^{17}$. 
No tocante aos gerentes, estes deverão adquirir um perfil de liderança situacional, cientes do seu papel de dirigir as atividades para desenvolver as pessoas e levá-las a realizar a contento, suas responsabilidades no atendimento à população, ao paciente e à família ${ }^{18}$.

A comunicação entre a equipe é de fundamental importância para que haja a interação e um real trabalho entre a equipe, descentralizada, levando os profissionais a se sentirem responsáveis pelas metas e tarefas para o êxito da ESF.

Compete ao enfermeiro ao liderar, conhecer o comportamento das pessoas que trabalham consigo e como acontecem as relações sociais e institucionais para poder identificar os problemas e necessidades da equipe e poder propor alternativas condizentes com a realidade que gerencia ${ }^{14}$.

Outro desafio das equipes é saber utilizar, de maneira mais adequada, os momentos de encontro com o usuário, quando o profissional, além de rea-lizar procedimentos técnicos, pode utilizar-se dessa situação para acolher demandas, formar vínculos e otimizar as relações humanas ${ }^{14}$.

O papel gerencial do enfermeiro recai na necessidade de construção e desenvolvimento, de forma articulada, das competências de natureza técnica, organizacional, comunicativa e sociopolítica. O profissional deve ter clareza de que essa construção extrapola um corpo de conhecimentos, na medida em que a responsabilidade e a atitude social devem ocorrer simultaneamente. Isso significa dizer que a atuação do profissional enfermeiro se expressa na competência humana para o cuidar, em suas múltiplas dimensões teórico-científica, social, política e, sobretudo, ética. O papel da avaliação para a tomada de decisão na gestão de serviços de saúde ${ }^{18}$.

$\mathrm{O}$ acolhimento deve ser visto como uma estratégia para conhecer e atender as reais deman 7 das/necessidades da população, ter como princípio atender a todas as pessoas que procuram os serviços de saúde; onde os profissionais devem escutar e dar uma resposta, capaz de resolver os problemas de saúde da população, bem como qualificar a relação trabalhador-usuário que deve dar-se por parâmetros humanitários de solidariedade e cidadania ${ }^{17}$.

As condições são desfavoráveis para o adequado desenvolvimento das atividades e do desempenho de suas competências, incluindo ambientes de trabalho inadequados, que favorecem o seu adoecimento ${ }^{17}$.

As dificuldades quanto aos recursos humanos são evidenciados no dia a dia relacionados com as diferentes personalidades e opiniões divergentes entre os membros da equipe devido a um despreparo dos ACS e do médico em integrar os princípios da estratégia e a dificuldade em articular uma assistência social para intervir nos problemas sociais da população 5 .

As dificuldades de trabalho na ESF não se limitam à organização multidisciplinar das atividades, mas, também, à sobrecarga de trabalho devido à grande demanda existente. Embora o Ministério da Saúde tenha estabelecido um número de atendimentos, a realidade de algumas equipes apontam demandas muito maiores daquilo que é preconizado, como se constatou nas respostas obtidas da pesquisa ${ }^{19}$.

Outra dificuldade evidenciada foi a falta de informação por parte da população quanto à finalidade da ESF, pois esta permanece na visão do modelo de saúde de assistência curativa, pontual, especializada e flexneriana ${ }^{5}$.

A falta de informação dos usuários priva-os de conhecer seus direitos e deveres de saúde. Observa-se que, quanto maior o nível de informação do usuário sobre os objetivos, e funcionamento da ESF, maior o grau de satisfação em relação ao programa, o que possibilita uma maior adesão. O nível de informação está condicionado, entre outros aspectos, pela eficácia das estratégias de comunicação e informação em saúde utilizada ${ }^{3}$.

Há ainda a dificuldade de compreender a referencia e a contrarreferencia. Sendo a atenção básica configurada como ordenadora do contato da população com o sistema de saúde, o trabalho deve ser organizado a partir da identificação das suas diversas necessidades de saúde, para que se responda de forma apropriada a elas e/ou para que se articule respostas em outros âmbitos, responsabilizando-se, com outros serviços e agentes, por sua resolutividade. É fundamental para a prática do enfermeiro estabelecer elos profissionais/institucionais, para que respostas mais efetivas à saúde de usuários sejam encaminhadas ${ }^{13}$.

Com relação à gestão, entende-se que o gerenciar envolve competências ${ }^{12}$, que incluem o relacionamento, 0 compromisso; a formação continuada; a educação permanente; a gestão participativa; a organização do serviço com um planejamento baseado nas necessidades locais, a definição de papéis e atribuições do pessoal, constituem-se como facilitadores do trabalho dos gerentes ${ }^{5}$.

A literatura aponta ainda que os enfermeiros enfrentam dificuldades no desenvolvimento da educação em saúde junto aos usuários, à equipe e aos gestores $(15,20)$ Já as dificuldades apontadas nos resultados da pesquisa, são consequências do perfil dos profissionais, da falta de recursos materiais, da estrutura física deficiente ${ }^{7}$. 
É importante a superação destas dificuldades considerando que o enfermeiro é reconhecido pela habilidade interativa e associativa, por compreender o ser humano como um todo, pela integralidade da assistência à saúde, pela capacidade de acolher e identificar-se com as necessidades e expectativas dos indivíduos, pela capacidade de interagir diretamente com 0 usuário e a comunidade, bem como pela capacidade de promover o diálogo entre os usuários e a equipe de saúde da família ${ }^{7}$.

\section{Considerações Finais}

A faixa etária dos enfermeiros estava entre 20 e 30 anos, ou seja, uma população jovem, a maioria tinha até um ano de trabalho na ESF e $12(80,0 \%)$ dos profissionais tinham Especialização na Saúde da Família.

Quanto às atribuições, desenvolvem ações propostas pela Política Nacional de Atenção Básica, como consultas de enfermagem, exame dePapanicolau, visita domiciliar, educação permanente para a equipe, ações junto à população, puericultura, gerenciamento em enfermagem, acolhimento, procedimentos técnicos (curativos, administração de medicamentos).

Entre as dificuldades mencionadas na execução do trabalho citaram a dificuldade para realização dessas atribuições: a estrutura física, o tamanho da população adscrita, os recursos materiais, humanos e financeiros, a gestão e a falta de informação por parte da população quanto à finalidade da ESF.

Esses dados permitiram concluir que há necessidade de se provocar nos enfermeiros uma reflexão acerca da necessidade de sua qualificação constante para melhor desenvolvimento de sua competência, nos processos gerenciais, em suas unidades.

A motivação da equipe, através do reconhecimento de suas ações refletirá na satisfação pessoal e consequentemente na maneira de tratar o usuário. A organização e planejamento das atividades, a definição das responsabilidades da equipe, através de uma gestão participativa é fundamental para a qualidade do trabalhado desenvolvido na ESF.

Constatou-se ainda a necessidade de melhorar o acesso à informação dos usuários sobre a finalidade da ESF e sobre a referência e contrarreferência, já que a falta dessa informação dificulta a relação do usuário com os serviços no SUS.

Além disso, são necessários de maiores investimentos nas ESF (recursos materiais, de estrutura física e humanos). A falta ou deficiência de infraestrutura comprometem a organização, o planejamento e a implementação das ações da ESF, interferindo de modo direto no trabalho da equipe e na assistência prestada aos usuários e ainda gerando desmotivação e desresponsabilização na execução das atividades.

Diante dos resultados obtidos, acredita-se que a atuação do enfermeiro na ESF é fundamental, porém, o profis $\urcorner$ sional precisa ter perfil e estar comprometido com a comunidade. Além disso, é fundamental o apoio e a participação dos demais profissionais, bem como do gestor, além dos investimentos necessários.

Destacam-se as limitações do estudo quanto ao próprio método. Sugerem outros estudos como novas metodologias para investigar, com o passar dos anos e a ampliação da cobertura os reflexos na atuação do enfermeiro na ESF, buscando-se evidenciar aspectos do saber-fazer do enfermeiro, tendo em vista acompanhar o modo como se configura a sua participação intersubjetiva nos rumos da atenção básica no país.

\section{Referências}

1. Soratto J, Pires DEP de, Dornelles S, Lorenzetti J. Family health strategy: a technological innovation in health. Texto Contexto - Enferm. 2015;24(2):584-92.

2. Campos GW de S, Carvalho YM de, Minayo MC de S, Junior MD, Akerman M. Tratado de Saúde Coletiva. 20 ed. Rio de Janeiro/RJ: Hucitec; 2012.

3. Shimizu HE, Carvalho Junior D de. O processo de trabalho na Estratégia Saúde da Família e suas repercussões no processo saúde-doença. Cien Saude Colet. 2012;17(9):2405-14.

4. Silva VG, Motta MCS, Zeitoune RCG. A prática do enfermeiro na Estratégia Saúde da Família: o caso do município de Vitória/ES. Rev Eletrônica Enferm. 2010;12(3):441-8.

5. Reis WG, Scherer MD dos A, Carcereri DL. O trabalho do Cirurgião-Dentista na Atenção Primária à Saúde: entre 0 prescrito e o real. Saúde Em Debate. 2015;39(104):56-64.

6. Silva SA, Oliveira F, Spinola CM, Poleto VC. Atividades desenvolvidas por enfermeiros no PSF e dificuldades em romper o modelo flexneriano. Rev Enferm Cent-Oeste Min. 2011;1(1):30-9. 
7. Backes DS, Backes MS, Erdmann AL, Büscher A. The role of the nurse in the Brazilian Unified Heath System: from community health to the family health strategy. Ciênc Saúde Coletiva. 2012;17(1):223-30.

8. Brasil. Resolução CNS n. 466, de 12 de dezembro de 2012. Estabelece as diretrizes e normas brasileiras regulamentadoras de pesquisas envolvendo seres humanos. 2012.

9. Brasil. Portaria no 2.488, de 21 de outubro de 2011. Aprova a Política Nacional de Atenção Básica, estabelecendo a revisão de diretrizes e normas para a organização da Atenção Básica, para a Estratégia Saúde da Família (ESF) e o Programa de Agentes Comunitários de Saúde (PACS). 2011.

10. Minayo MC de S. Pesquisa social : teoria, método e criatividade. 230 ed. Rio de Janeiro: Vozes; 2012.

11. Andrade AM, Guimarães AMDN, Costa DM, Machado L de C, Gois CFL. Visita domiciliar: validação de um instrumento para registro e acompanhamento dos indivíduos e das famílias. Epidemiol E Serviços Saúde. 2014;23(1):165-75.

12. Meira MDD, Kurcgant P. College Program evaluation according to graduates. Rev Esc Enferm USP. 2009;43(2):481-5.

13. Silva TAM da, Fracolli LA, Chiesa AM. Professional trajectory in the Family Health Strategy: focus on the contribution of specialization programs. Rev Lat Am Enfermagem. 2011;19(1):148-55.

14. Souza MG, Mandu ENT, Elias AN. Perceptions of nurses regarding their work in the Family Health Strategy. Texto Contexto - Enferm. 2013;22(3):772-9.

15. Silva ANB da, Franco ES, Neto EMR, Coelho MO, Bezerra JCP. Elaboração de material didático para educação em saúde direcionado para hipertensão arterial. Saúde St Maria. 2015;41(1):175-84.

16. Versian CC, Fonseca MG, Bretas TCS, Andrade FM, Rocha ALF. Significado da consulta de enfermagem às crianças menores de dois anos na percepção das mães. Enferm Em Foco. 2014;4(2):123-6.

17. Trindade L de L, Peron A, Amestoy SC, Gehlen GC, Noguez PT. Reflexões acerca do perfil de atendimento na Estratégia Saúde da Família. Cogitare Enferm. 2011;16(1):162-6.

18. Tanaka OY, Tamaki EM. O papel da avaliação para a tomada de decisão na gestão de serviços de saúde. Ciênc Saúde Coletiva. 2012;17(4):821-8.

19. Rangel RF, Fugali M de M, Backes DS, Gehlen MH, Souza MHT. Avanços e perspectivas da atuação do Enfermeiro em Estratégia Saúde da Familia. Cogitare Enferm. 2011;16(3):498-504.

20. Roecker S, Budó M de LD, Marcon SS. The educational work of nurses in the Family Health Strategy: difficulties and perspectives on change. Rev Esc Enferm USP. 2012;46(3):641-9.

\title{
Cristiane Aparecida Silveira
}

Endereço para correspondência - Rua: Av. Pe. Francis Cletus Cox, n 1661, Bairro: Jardim Country Club, CEP: 37701355, Poços de Caldas, MG, Brasil.

E-mail: casilve@yahoo.com.br

Lattes: http://lattes.cnpq.br/8840798305145545

Anilson Augusto Firmino - augustofirmino1@hotmail.com

Mariana Cristina Moraes - mariana_crismoraes@yahoo.com.br

Priscila Elídia Alves Nascimento - priscila_ean@hotmail.com

Sônia Maria Alves de Paiva - paiva@pucpcaldas.br

\author{
Enviado em 07 de julho de 2015. \\ Aceito em 13 de janeiro de 2016.
}


